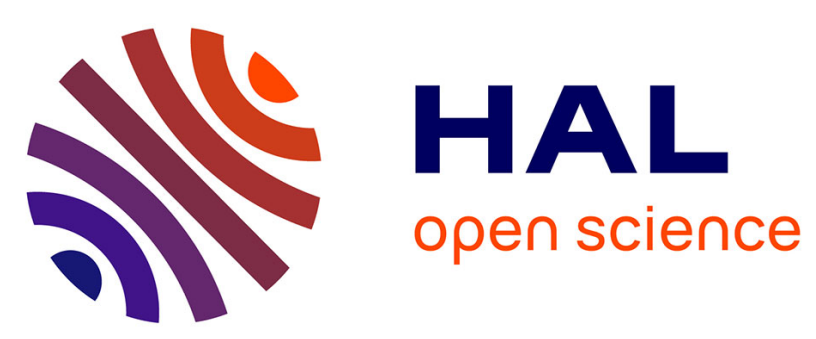

\title{
A novel indoor localization scheme based on fingerprinting technique And CDMA signals
}

Nadia Aloui, Kosai Raoof, Ammar Bouallegue, Stephane Letourneur, Sonia Zaibi

\section{- To cite this version:}

Nadia Aloui, Kosai Raoof, Ammar Bouallegue, Stephane Letourneur, Sonia Zaibi. A novel indoor localization scheme based on fingerprinting technique And CDMA signals. IPIN 2012 - 3rd International Conference on Indoor Positioning and Indoor Navigation, Nov 2012, Sydney, Australia. pp.1 5, 10.1109/IPIN.2012.6418941 . hal-01099467

\section{HAL Id: hal-01099467 https://hal.science/hal-01099467}

Submitted on 3 Jan 2015

HAL is a multi-disciplinary open access archive for the deposit and dissemination of scientific research documents, whether they are published or not. The documents may come from teaching and research institutions in France or abroad, or from public or private research centers.
L'archive ouverte pluridisciplinaire HAL, est destinée au dépôt et à la diffusion de documents scientifiques de niveau recherche, publiés ou non, émanant des établissements d'enseignement et de recherche français ou étrangers, des laboratoires publics ou privés. 


\title{
A Novel Indoor Localization Scheme Based On Fingerprinting Technique And CDMA Signals
}

\author{
Nadia ALOUI* ${ }^{* \dagger}$ Kosai RAOOF ${ }^{\ddagger}$, Ammar BOUALLEGUE $^{\dagger}$, Stephane LETOURNEUR ${ }^{\ddagger}$ and Sonia ZAIBI \\ ${ }^{*}$ Grenoble University, GIPSA-Lab, France \\ †ENIT School, 6’Com-Lab, Tunisia

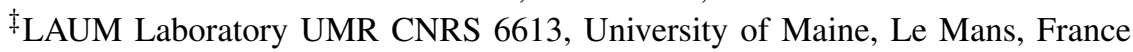 \\ Email: nadia.aloui@gipsa-lab.grenoble-inp.fr,kosai.raoof@univ-lemans.fr,ammar.bouallegue@enit.rnu.tn, \\ stephane.letourneur@univ-lemans.fr,Sonia.Zaibi@enit.rnu.tn
}

\begin{abstract}
In this paper, we propose a novel acoustic localization system based on fingerprinting technique. It deploys the Time Of Arrival of CDMA signals emitted by speakers to locate a microphone. The system is inspired from our earlier proposed scheme [1] which deploys the lateration method. Here, we adopt the fingerprinting technique since it is more applicable to indoor environments. The position estimation is accomplished through nonparametric kernel regression. Performance are evaluated by experiments, performed in a hall of interns in National School of Engineers of Le Mans. Results have shown that our proposed scheme provides an accuracy of $8.5 \mathrm{~cm}$ within $80 \%$ precision.
\end{abstract}

Keywords : Acoustic localization, fingerprinting technique, nonparametric kernel regression, time of arrival, CDMA signals.

\section{INTRODUCTION}

Indoor positioning is an important technique due to its useful and various applications, such as, tracking the doctors and patients in hospitals and museum tour-guide.

The localization technique could be mainly classified into two categories: triangulation and fingerprinting technique.

Triangulation combines angulation and lateration. In the case of the angulation, the position of an object is estimated by measuring the angle to it from at least two known locations. For the lateration method, the position is estimated by measuring its distances from at least three references of known locations. Both angulation and lateration need the line of sight between the transmitter and the receiver. However, this assumption is not always available in indoor environments because of the presence of obstacles.

Fingerprinting technique is widely suitable for indoor localization. It includes two phases. First, a set of sampling locations is selected in the target environment, then, a locationdependent signal parameters is measured at each location. Second, the target position is estimated by comparing its signature with the previously built database through pattern matching algorithms.

The most widely used localization system is the Global Positioning System (GPS). Although, it allows good performance in outdoor environment, it cannot be deployed in indoor environment. For indoors, several researches have been carried out and many location systems have been proposed. Based on the type of the emitted signal, indoor systems can be classified into Infrared-based positioning system, Radiofrequency and Ultrasonic systems.

\section{- Infrared Positioning system}

The IR-based positioning systems are the most common positioning systems due to the availability of IR technology. An IR positioning system requires line-of-sight communication between emitters and receivers and provides accurate positioning estimations. An example of an IR-based indoor location system is the Active Badge system. An active badge, attached to a person, emits a unique IR signal every 15 seconds [3]. Sensors are fixed in each located place to detect the emitted signal and forward the location information to a central server, in order to locate the active badge. The IRbased Positioning system has a simple architecture. Therefore, it requires no much time for its installation or maintenance. However, the IR signals are sensitive to interference from florescent light and sunlight [2]. A solution of such problem is to use optical and electronic filters [4] and to implement noise cancelling algorithm at the receivers [2], which raise the system cost. Moreover, the IR-based Positioning system hardware is expensive.

\section{- Radio Frequency Positioning Systems}

The radio frequency positioning systems allow a large coverage area due to the ability of radio waves to pass through walls. The systems mainly use triangulation and fingerprinting techniques and employ the existing RF infrastructures like the WLAN. The RFID positioning system includes the RFID tags and RFID reader. We distinguish two types of tags: passive and active tags [9]-[10]. A passive tag is a receiver without battery. It's small and cheap. An active tag is a small transceiver. It's more expensive and supports higher range. The RFID reader determines, among different levels, the level of the received power. Based on the RSS data, the RFID systems estimate an object position with a low accuracy.

- US Systems

The active Bat system [5] allows 3-D position of a tag carried by a person using the UltraSonic technology. The tag emits periodically a short pulse of ultrasound. Its distances from at least 3 receivers, fixed on the ceiling, are measured. Then, the tag position is calculated based on multilateration technique [6]. 
In the cricket system [7]-[8], emitters of known positions emit RF and US signals. A receiver mounted on the object to be located measures the TOA of US signals and uses the triangulation technique to estimate its position. RF signals are transmitted for synchronization of TOA measurement. Similarly to GPS, the Dolphin system deploys DS-CDMA method to obtain simultaneous distance measurements. The multilateration technique is adopted for the position estimation using Time of Flight measurement. Two systems have been proposed depending on whether the object to be localized is the emitter or the receiver. The accuracy of the polled and centralized system (the system where the object is the emitter) is about $2 \mathrm{~cm}$, while, the privacy-oriented location system gives an accuracy of $4.9 \mathrm{~cm}$ for synchronous case and an accuracy of $26.6 \mathrm{~cm}$ for asynchronous case.

Our earlier proposed scheme [1] is similar to the Dolphin system, except that it adopts trilateration instead of multilateration. Given four tweeters, two methods of selecting available distance measurements have been proposed to locate a microphone.

- Method 1

The method 1 utilizes each set of three measured distance to estimate the position. The location estimate corresponds to the center point of the four estimates.

\section{- Method 2}

The method 2 eliminates the least reliable distance corresponding to the smallest correlation peak and uses only the three most reliable measured distances for the position estimate. The system has $2 \mathrm{~cm}$ accuracy.

In this paper, we propose a novel localization system that deploys three tweeters and that is more suitable to indoor environment. It is based on fingerprinting technique and utilizes the local linear estimator as a matching algorithm. The system is privacy-oriented and can utilize cheap equipment.

The rest of paper is organized as follows. In section II, a description of the proposed localized system is provided. The measurement system and setup are presented in section III. Section IV gives the results and discussion. Finally, we conclude the paper and point out future directions in section $\mathrm{V}$.

\section{PRoposed LOCATION SYSTEM}

One possible scenario of the proposed system is given as follows:

- An object wishing to estimate its position send a request to a server,

- The tweeters emit, then, known acoustic signals,

- The object records the received signal and treats it to get its location.

In this paper, we focus on the processing phase that gives the estimate position.

\section{A. Distance measurement}

The proposed location system deploys DS-CDMA signals to locate a microphone. The acoustic signals were chosen due to their low velocity.

The spread spectrum technique is used because it has several advantages. First, the technique is resistant to narrow-band interference. Second, the spread spectrum sequences have a low cross-correlation value, which, allows multiple devices to broadcast in the same frequency range. Third, they have good auto-correlation properties enabling code synchronization. Therefore, these codes allow accurate and simultaneous time measurement. The m-sequences and the orthogonal codes are not suitable to our system. In fact, The $\mathrm{m}$-sequences are not generated in a large number and the orthogonal codes are used when times of arrivals of the signal to the receiver are the same. Gold codes were selected thanks to their advantage in generating large numbers of codes and their good autocorrelation and cross-correlation properties.

Three tweeters, identified each by a unique code, are deployed. The BPSK modulated signals are emitted simultaneously and the microphone receives the aggregate data signal. The received signal is correlated with the tweeters' codes. The time of the peak correlation value for each correlation code, expressed in terms of sampling periods $L$, is selected. It gives an estimate of the Time of arrival of signal between the microphone and the corresponding speaker. Hence, the measured distances are deduced from the following formula:

$$
d=\frac{L * v_{\text {sound }}}{f_{\text {sampling }}}
$$

where

- $v_{\text {sound }}$ : is the velocity of the sound and

- $f_{\text {sampling: }}$ is the sampling frequency.

The three measured distances are concatenated to form the signature of the microphone location. Note that the times of arrivals of the signal to the microphone can also form the signature.

\section{B. Local linear estimator}

Let number of fingerprint locations be $L, s$ the fingerprint of the object to be localized and $s_{l}$ the fingerprints at positions $c_{l}(l=1,, L)$.

Based on the previous data, we aim to estimate the object position $c$. This means the conditional expectation $\tilde{c}=E(c \mid s)$, denoted by $m(s)$, and defined as

$$
E(c \mid s)=\int \frac{c * f(c, s) d c}{f(c, s) d c}
$$

where $f(c, s)$ is the joint probability density function.

To explain the principle of the local linear estimator, we consider the first order Taylor expansion of the unknown conditional expectation $m($.$) :$

$$
m(t)=m(x)+m^{(1)}(x) *(t-x)(3)
$$

for $t$ in a neighborhood of a point $x$. The minimization problem is expressed as:

$$
\min _{\beta} \sum_{i=1}^{L}\left(c_{i}-\right.
$$


$\left.\beta_{0}-\beta_{1} *\left(s_{i}-s\right)^{2}\right) * K_{H}\left(s_{i}-s\right)(4)$

Where $\beta=\left(\beta_{0}, \beta_{1}\right)^{T}$ the coefficients vector and $K_{H}\left(s_{i}-s\right)$ the kernel weight that realize the neighborhood and is defined as:

$$
K_{H}(.)=\frac{1}{\operatorname{det}(H)} * K\left(H^{-1}(.)\right)
$$

The function $K$ is the kernel function and the matrix $H$ is known as the bandwidth matrix.

The solution of (4) is, then, a weighted least squares estimator given by:

$$
\tilde{\beta}=\left(S^{T} W S\right)^{-1}\left(S^{T} W C\right)
$$

Where

$$
\begin{gathered}
C=\left|\begin{array}{c}
c_{1} \\
\vdots \\
c_{L}
\end{array}\right| \\
S=\left|\begin{array}{ll}
1 & \left(s_{1}-s\right)^{T} \\
\vdots & \vdots \\
1 & \left(s_{L}-s\right)^{T}
\end{array}\right|
\end{gathered}
$$

And

$$
W=\operatorname{diag}\left(K_{H}\left(s_{1}-s\right), \ldots, K_{H}\left(s_{L}-s\right)\right)
$$

Therefore, the local linear estimator of the position $c$ is:

$$
\tilde{c}=\beta_{0}
$$

The shape and orientation of the kernel function $K$ is controlled by the bandwidth matrix $H$. In this paper, we adopt the Gaussian kernel function:

$$
K(y-z)=\frac{1}{(2 \pi)^{\frac{N}{2}}} \exp \left[\frac{-1}{2}(y-z)^{T} *(y-z)\right]
$$

The value of $K(y-z)$ is larger when $y$ is closer to $z$. We use the multivariate generalization of the Scott's Rule of Thumb for bandwidth selection that derives the matrix $H$ as [12]:

$$
H=L^{\frac{-1}{N+4}} * C o
$$

Where $C o$ is the sample covariance matrix of the fingerprint vectors $s_{1}, s_{2}, \ldots ., s_{L}$. Note that this rule takes into account the statistical dependence between the signature vector elements.

The position estimate $\tilde{c}$ given by the local linear estimator (10) can be equivalently written as [11]:

$$
\tilde{c}=\frac{T_{0}-S_{1}^{T} S_{2}^{-1} T_{1}}{S_{0}-S_{1}^{T} S_{2}^{-1} S_{1}}
$$

Using the notations

$$
S_{0}=\sum_{i=1}^{L} K_{H}\left(s_{i}-s\right)(14)
$$

$$
\begin{gathered}
S_{1}=\sum_{i=1}^{L} K_{H}\left(s_{i}-s\right)\left(s_{i}-s\right)(15) \\
S_{2}=\sum_{i=1}^{L} K_{H}\left(s_{i}-s\right)\left(s_{i}-s\right)\left(s_{i}-s\right)^{T}(16) \\
T_{0}=\sum_{i=1}^{L} K_{H}\left(s_{i}-s\right) c_{i}(17) \\
T_{1}=\sum_{i=1}^{L} K_{H}\left(s_{i}-s\right)\left(s_{i}-s\right) c_{i}(18)
\end{gathered}
$$

Note that $S_{1}$ and $T_{1}$ are $N$-variate vectors and $S_{2}$ is $N * N$ matrix.

\section{Measurement System And SetuP}

\section{A. Testbed}

Our testbed was located on the hall of interns in National School of Engineers of Le Mans. The hall, shown in figure (1), consists of computer areas, desks and different materials Figure (2) shows the effective area where we perform the experiment. It measures $1.5 \mathrm{~m} * 1.5 \mathrm{~m} * 0.75 \mathrm{~m}$. The experiment setup can be shown in figure (3).

Unlike the testbed built in our earlier proposed localization scheme [1], no arrangement has been taken, here, to reduce the effects of reflections and exterior influences. Therefore, the proposed system is more suitable to indoor environment. Recalling that in [1], the testbed was encircled with curtain. The ceiling was covered with a material of low reflectivity and the floor was covered with carpet.

As [1], the system can locate simultaneously more than one microphone.

\section{B. Material}

The hardware system is composed of a pc, a signal acquisition module, an amplifier, three tweeters and one microphone. The signal acquisition module deploys three uniform output channels to broadcast the three signals produced by the pc, and, one uniform input channel to record the aggregate signal received by the microphone. Lab view is utilized to perform the data acquisition between the $\mathrm{pc}$ and the signal acquisition module.

\section{RESUlTS}

Performance of the proposed method are evaluated in terms of accuracy and precision. Accuracy is defined as the highest tolerated error and the precision is the ratio of the measurements with errors equal to or less than the accuracy. The system variables are fixed to:

- $51200 \mathrm{~Hz}$ for sampling frequency,

- $3000 \mathrm{~Hz}$ for carrier and chip frequency,

- 127bits for length of Gold code sequence.

For the off-line phase, we collect the signatures of 25 sampling locations separated by $30 \mathrm{~cm}$. For the on-line phase, we collect another set of signatures in 29 positions, randomly distributed in the effective area. Localization was attempted 
(a)

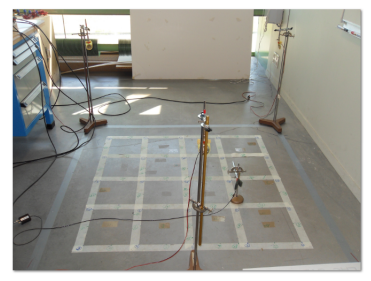

(b)

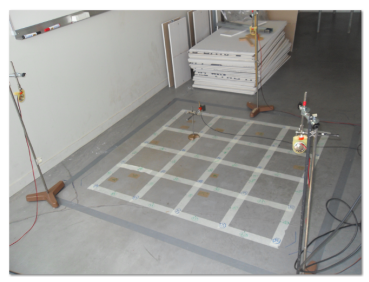

(c)

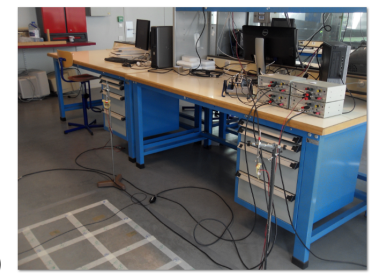

Fig. 1: Measurement environment- (a) side 1, (b) side 2, (c) side 3

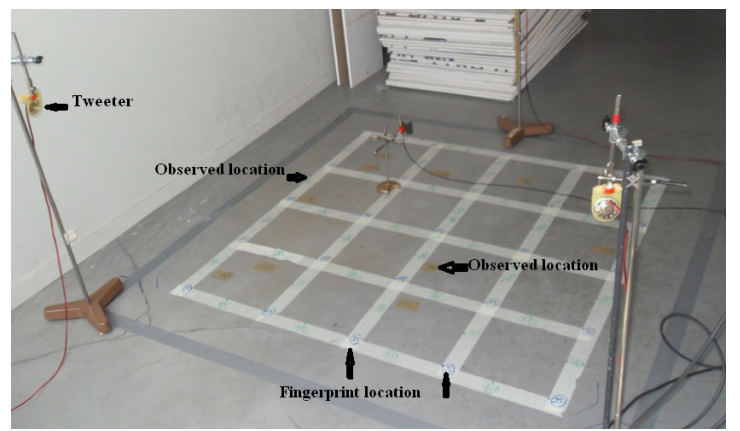

Fig. 2: Marks of fingerprint location, observed positions and tweeters

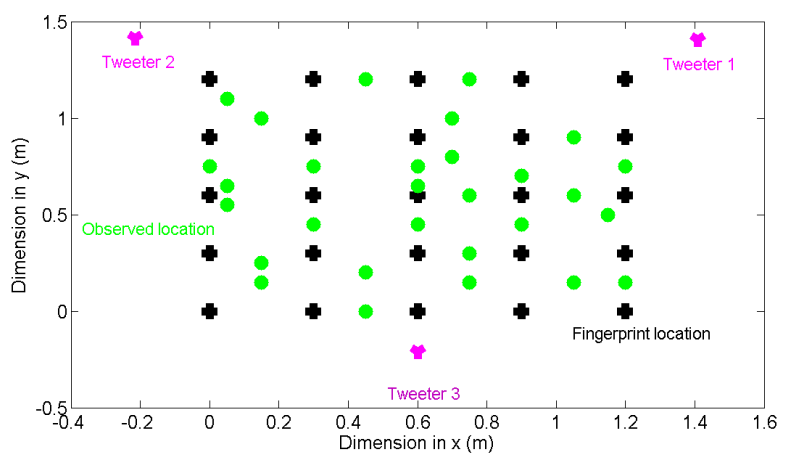

Fig. 3: The layout of the experiment setup

TABLE I: Positioning accuracy and precision obtained with the proposed scheme

\begin{tabular}{|c|c|c|c|c|c|}
\hline Error distance(meters) & 0.05 & 0.06 & 0.085 & 0.12 & 0.15 \\
\hline Precision Percentage (\%) & 55.66 & 66.50 & 80.29 & 90.64 & 94.08 \\
\hline
\end{tabular}

several times at each position.

In figure (4), the percentage of realizations was depicted as a function of the location estimation accuracy. The proposed system provides accuracy about $8.5 \mathrm{~cm}$ almost $80 \%$ of time. The mean error location computed for all positions is about $6.5 \mathrm{~cm}$. This value is achieved within $68.5 \%$ precision.
If we compare the described system to our earlier proposed system [1], it is seen that the earlier proposed system [1] gives an accuracy of $2 \mathrm{~cm}$ within $99 \%$ precision, while the proposed system provides $8.5 \mathrm{~cm}$ accuracy within $80 \%$ precision. However, it is worth mentioning that in [1] several arrangements have been considered, in the test-bed, to assure reducing the effects of exterior influences and multipath disturbances due to the reflections. Here, the test-bed is a part of a hall full of different types of material, desks, computers, and it was not modified in order to evaluate the performance of our system.

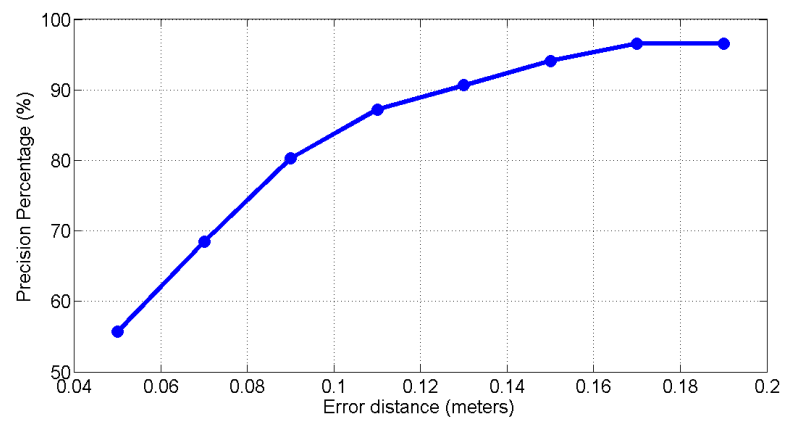

Fig. 4: Precision versus location estimation accuracy

\section{CONClusion}

In this paper, we have proposed a novel acoustic localization system based on fingerprinting technique. The Time Of Arrival of CDMA signals emitted by speakers are deployed to locate a microphone. The system is more suitable to indoor environment than that described in [1].

Compared to systems employing Ultrasonic waves or Infrared signals, it is seen that these systems usually provide a better ranging accuracy than those based on audible sound. However, for the IR-based systems the required equipments are expensive. For the US systems, a dedicated infrastructure is needed. The advantage of the audible positioning systems is that nowadays, various mobile devices are equipped with speaker and microphone. Therefore, users can utilize their devices in an audible sound positioning system to estimate their positions.

We suggest several future directions based on this work. First, we aim to evaluate the performance of the system in an extended test-bed. Second, we would like to study the performance of the method when the effective area contains different voice sources. Third, our work in this paper has been limited to the localization in 2D coordinate system. We would want to extend the work to 3D coordinate system.

\section{ACKNOWLEDGEMENT}

The authors would like to thank the anonymous reviewers for their valuable comments and suggestions. We wish also to thank M. Mathieu Secail-Geraud for his collaboration. 


\section{REFERENCES}

[1] Cem Sertatila, Mustafa A. Altinkayaa, Kosai Raoof, A Novel Acoustic Indoor Localization System Employing CDMA, Digital Signal Processing 22 (2012) 506-517.

[2] X. Fernando, S. Krishnan, H. Sun, and K. Kazemi-Moud, Adaptive denoising at Infrared wireless receivers, Proc. SPIE, 2003

[3] R. Want, A. Hopper, V. Falcao, J. Gibbons, The Active Badge Location System, ACM Trans. Information Systems, vol. 10, no. 1, January 1992, pp. 91-102.

[4] C. Lee, Y. Chang, G. Park, J. Ryu, S. Jeong, and S. Park, Indoor Positioning System Based on Incident Angles of Infrared Emitters, Industrial Electronics Society, 2004.

[5] Active Bat website, 2008, http://www.cl.cam.ac.uk/research/dtg/attarchive/bat/

[6] A. Ward, A. Jones, and A. Hopper, A New Location Technique for the Active Office, IEEE Personal Communications, vol. 4, no. 5, October 1997, pp. 42-47.

[7] N. Priyantha, A. Chakraborty, and H. Balakrishnan, The cricket locationsupport system, Proc. ACM Conference on Mobile Computing and Networking, 2000.

[8] N. B. Priyantha, The Cricket Indoor Location System, PhD thesis, MIT, 2005.

[9] L. M. Ni and Y. Liu, LANDMARC: Indoor Location Sensing Using Active $R F I D$, Proc. IEEE International Conference on Pervasive Computing and Communications, 2003, pp. 407-416.

[10] K. Finkenzeller, RFID-Handbuch, Hanser Fachbuch, 1999. Also available in English as RFID Handbook: Radio-Frequency Identification Fundamentals and Applications, John Wiley and Sons, 2000.

[11] W.Hrdle and M.Mller,Multivariate and Semiparametric Kernel Regression, To appear in M.G. Schimek (Ed,), Smoothing and regresion. Approaches, Computation and Applications, 1996.

[12] W.Hrdle, M.Mller, Stefan Sperlich and A. Werwatz,Nonparametric and Semiparametric Models

Y.Jin, W.Soh and W.Wong Indoor Localization with Channel Impulse Response Based Fingerprint and Nonparametric Regression 